\title{
Research of current studying status and developing trend of decoupling algorithm for six-axis force/torque sensor
}

\author{
Binbin Han, Yingjun $\mathrm{Li}^{\mathrm{a}}$, Guicong Wang \\ School of Mechanical Engineering, University of Jinan, Jinan 250022, China
}

\begin{abstract}
For different structures of sensor have many decoupling algorithms, this paper discusses mainly from two aspects the decoupling algorithm of six-axis force/torque sensor. One is the linear decoupling which is under the assumption that the sensor system is a linear system, another is the nonlinear decoupling which is under the assumption that the sensor system is a nonlinear system. The paper also analyzes and compares several commonly used decoupling algorithms of six-axis force/torque sensor from the aspects of decoupling effects and the accuracy of measurement, thus providing some academic bases and references for the further research of decoupling algorithm. Besides, taking the appropriate and effective decoupling algorithm can improve the measuring accuracy of the sensor to the largest extent, and meet the requirements for sensors reaching high-level, precise and sophisticated performances. Finally, the decoupling algorithms for six-axis force/torque sensor are summarized and its research direction can be foretold.
\end{abstract}

Keywords: six-axis force sensor; decoupling algorithm; genetic algorithm; neural network.

\section{Introduction}

Sensor, communication and computer technology are known as the three pillar industries of modern information technology. They play a role in the sensing, transmission and processing of the measured information. The sensor technology, as a new and high technology industry, is recognized with the best prospects at home and abroad, and as a symbol of the advanced development in the modern industry.

Six-axis force/torque sensor can measure all the forces of information in the space (three orthogonal forces and three orthogonal moments) and force point location information [1]. It is the most important sensor of sensors and widely used in robot, precision assembly, aerospace, mechanical processing and automobile, etc [2]. Due to the wide use of the sensor, many researchers have proposed and designed a variety of structural forms of six-axis force sensor. Watson proposed parallel structure six-axis force sensor [3]. Nguyen and Charles proposed Stewart structure six-axis force sensor[4]. Yoshikawa put forward the non-radial structure of six-axis force sensor [5]. Due to factors such as structure design, manufacture, installation process, when six-axis force sensor is working, there will always be different degrees of dimensional coupling among the most sensor output signals. Dimensional coupling will reduce the accuracy of the sensor and restrict the application of sensors in the high-level, refined, sophisticated field, etc. Therefore, we must eliminate or restrict the sensor coupling between dimensions

\footnotetext{
${ }^{a}$ Corresponding author : me_liyj@ujn.edu.cn
} 
in order to improve the accuracy of the sensor. The key of research six-axis force/torque sensor is to eliminate the dimensional coupling errors as much as possible[6]. Thus, the research of the decoupling algorithm has become a hot topic for many scholars.

\section{Current study status of decoupling algorithms for six-axis force/torque sensor}

There are two main types of coupling causes for errors of six-axis force/torque sensor: structural coupling and error coupling[7]. Structural coupling, which is due to the defects in the design of the structure, lead to the coupling between mutual dimensions in the process of sensor design. Error coupling is caused by patch error and installation process with almost inevitable effects. At present, the decoupling algorithm mainly taken is to compensate the output of the sensor. To decouple the sensor means that take the ways of compensation calculation to reduce coupling for the error coupling. At this stage of decoupling algorithm for sensor mainly includes static linear decoupling algorithm and static nonlinear decoupling algorithm. Here are several kinds of representative ones.

\subsection{The linear decoupling algorithm for six-axis force/torque sensor}

Under the assumption that the sensor is a linear output system, linear decoupling is used through the establishment of the linear relationship between the sensor output channel and the linear relationship between six-axis force. Currently there are two main types of linear decoupling methods: one is static decoupling based on the traditional linear calibration; the other is based on the least squares.

\subsection{The traditional static linear decoupling}

The traditional static linear decoupling algorithm of six-axis force/torque sensor is based on linear calibration. Assume that the sensor is a linear system, we can determine the calibration matrix based on the six-axis force sensor calibration experiments in all directions [8].

C. Yuan took the traditional static calibration method for the six-axis force/torque sensor with the cross beam structure [9]. The experimental results proved that he designed the sensor with sufficient sensitivity, with linear errors being less than $0.62 \% \mathrm{~F}$.S, lag errors being less than $0.73 \% \mathrm{~F}$.S, repeatability being less than $1.88 \% \mathrm{~F}$.S and the interference error being less than $3.0 \% \mathrm{~F}$.S. It is suitable to be used in a humanoid robot feet.

\subsection{Linear decoupling based on least square method}

Y. Sun designed a six-axis force/torque sensor, by using the least squares method to decouple the experimental data [10]. The decoupled sensor has good linearity, repeatability, stability, sensitivity and accuracy. The experimental results proved that the sensor can meet the requirements of space robot.

\subsection{Other linear decoupling algorithm}

C. Mao proposed a static decoupling algorithm based on coupling error modeling on the basis of the least square algorithm [11]. The experimental data are calculated by using subsection two order fitting method. Experiments proved that the algorithm is more stable than the decoupling algorithm of least square method, and the precision of the sensor is improved.

Because the sensor is not a linear dimensional coupling system, so the linear decoupling algorithm has become increasingly inadequate in sensor applications. Decoupled sensor has characteristics like low precision, big error and the decoupling effect which are not ideal. So that the sensor can not meet the high performance requirements. Nonlinear decoupling algorithm shows a trend of diversification and the 
system model of the sensor is similar to the nonlinear, so it can vividly expressed the output of the sensor by using the nonlinear decoupling algorithm. Therefore, further studies need to be carried out on the nonlinear decoupling algorithm of sensor.

\subsection{The nonlinear decoupling algorithm for six-axis force/torque sensor}

The nonlinear model can realistically reflect the actual output of multi-axis force / torque sensor and completely solve the sensor static coupling problem in theory when the nonlinear decoupling of the sensor is adopted [12].

\subsection{Decoupling algorithm based on bp neural network}

Q. Liang analysed and decoupled the E type ultrathin film strain six-axis force/torque sensor [13]. Train and test the neural network model of sensor by using BP error back propagation algorithm based on MATLAB. The experimental results proved that the sensor has high linearity and isotropic after decoupling, with the maximum of class I error being only $0.17 \%$ and the maximum of class II error being only $1.6 \%$. With the characteristics like high sensitivity, linearity, weak dimensional coupling, it can basically meet the requirements of the sensor performance index.

\subsection{Decoupling algorithm based on support vector machine (SVM)}

Y.K. Ma proposed the method that is to composite calibration the multi-axis force sensor by the method of SVM and studied the six-axis force sensor with cross beam structure[14]. This method solved the coupled nonlinear error of multi-axis force sensor and verified that it can improve the measurement accuracy of the sensor in the six-axis force sensor calibration experimen and have better universal applicability.

\subsection{Decoupling algorithm based on radial basis function (RBF)}

W.B. Xiao compared the Cramer rule with the linear decoupling algorithm of the least square method and proposed a nonlinear decoupling algorithm model by using RBF radial neural network based on the analysis of the linear decoupling principle of multi-axis force sensor[15]. He summed that the calibration matrix rule got from the least square method has better isotropic than the Cramer, and the inter dimensional error and coupling can be greatly reduced with RBF approximation function relation between output and input.

\subsection{The hybrid hierarchy genetic algorithm and neural network hybrid algorithm}

Z.P. Shi studied the large range 6-UPUR structure of six-axis force sensor nonlinear decoupling [16]. He proposed three nonlinear static decoupling methods based on neural network optimized by hybrid hierarchy genetic algorithm: HHGA-BP network algorithm, HHGA-RBF network algorithm and HHGA-WNN algorithm. Experimental results showed that the three kind of hybrid genetic algorithm is better than single BP network and RBF network algorithm and dimensional coupling is smaller. The use of this method has greatly improved the measurement accuracy of six-axis force sensor. The class I errors and class II errors are less than 3\%. It provides a way of optimization for the future research on static nonlinear decoupling of six-axis force sensor. 


\subsection{Other nonlinear decoupling algorithm}

F. Chen overcame the shortcomings of traditional static decoupling method on the six-axis wrist force sensor [17]. The static nonlinear forward decoupling algorithm is constructed by polynomial nonlinear approximation, which avoids the non practicality of the curve fitting and the inverse solution in the traditional algorithm. The equations in the algorithm can be applied to both linear and nonlinear systems and have a wide range of extended space. Experimental results proved that this method is better than the traditional static linear decoupling method.

\section{The development direction of the six-axis force/torque sensor decoupling algorithm}

Along with the constant innovation of sensor technology, sensor decoupling algorithm is increasingly becoming the focus of research. Six-axis force/torque sensor took the new algorithm based on the fusion of a variety of algorithms with advantages of good decoupling effects, the low coupling coefficient of the sensor and the high measurement precision and so on. In addition, scholars still stay in the static decoupling stage for the sensors decoupling research. If only the sensor can be dynamically decoupled, can the real-time dynamic characteristics of the sensor be realized. In view of the above decoupling characteristics, we can propose the following idea: to establish a real time and dynamic self decoupling calibration system based on the fusion of a variety of algorithms for the sensor. At the same time, through optimizing the structure of sensor and studying out a type of sensor that can directly output self decoupling six-axis force/torque sensor, it can completely eliminate or restrain the sensor coupling in theory and make the measurement accuracy of the sensor to reach the optimum, and meet the requirements of the development in high-tech in the field of sensor. This assumption provides a reference direction for the subsequent research of the decoupling algorithm, and will become a new trend in the future.

\section{Conclusions}

This paper elaborated the current study status and developing trend of decoupling algorithm for six-axis force/torque sensor at home and abroad. It mainly reviewed the two aspects of static linear decoupling algorithms and nonlinear decoupling algorithms for six-axis force/torque sensor. At present, because of the strain sensor technology is quite mature, there are more researches on the decoupling algorithm of strain six-axis force/torque sensor. On the other hand, although the research on the decoupling algorithm of piezoelectric and magnetoelastic sensor is comparatively less, the decoupling algorithm of strain sensor is applicable to these sensors according to the detection principle and decoupling method. This paper provided a theoretical basis for further research on six-axis force/torque sensor decoupling algorithm and provided an insight into the future Six-axis force/torque sensor decoupling algorithm.

\section{Acknowledgements}

This work is supported by the National Natural Science Foundation of China (51205165) and Shandong Provincial Natural Science Foundation Joint project of China (2015ZRB01240).

\section{References}

1. M. Li, J. Liu, L. Qin, et al., JOURNAL OF MECHANICAL ENGINEERING, Vol. 6 (2014) 1-7, In Chinese.

2. Y.L. Hou, K.J. Kang, D.X. Zeng, et al., Journal of Optics and Precision Engineering, Vol.7(2008)1258-1265, In Chinese. 
3. Watson,C. Paul, Drake and L.L. Samuel, U.S. Patent 4,094,192. (1978)

4. C.Charles, Nguyen, C. Sami, Antrazi, et al., Computers \& Electrical Engineering, Vol. 17 (1991) 191-203.

5. T. Yoshikawa, T. Miyazaki, A six-axis force sensor with three-dimension cross-shape structure, in IEEE International Conference on Robotics and Automation,(Scottsdale, USA, 1989).

6. J.Q. Ma, A.G. Song, Development of a Novel Two-Axis Force Sensor for Chinese Massage Robot, Advances in Precision lnstrumentation and Measurement,Vol.13 (2012), pp.299-304.

7. H.B. Cao, Y.X. Sun, et al., CHINESE JOURNAL OF SENSORS AND ACTUATORS, Vol. 24 (2011) 1136-1140, In Chinese.

8. J.Z. Zhang, K. Guo, C. Xu, Transducer and Microsystem Technologies, Vol. 12 (2007), pp.58-59, In Chinese.

9. $\quad$ C. Yuan, et al., Measurement, Vol. 70 (2015) 110-122.

10. Y. Sun, et al., Measurement, Vol. 44 (2014) .135-148, In Chinese.

11. C. Mao, A.G. Song, X. Gao, et al., CHINESE JOURNAL OF SENSORS AND ACTUATORS, Vol. 2 (2015) 205-210, In Chinese.

12. H. Cao, Y. Yu, Y. Ge, A research of multi-axis force sensor static decoupling method based on neural network, Proceeding of the IEEE International conference on Automation and Logistics, (Washington, DC,USA, 2009).

13. Q. Liang, et al., Measurement, Vol. 43 (2010) 1702-1719.

14. Y.K. Ma, X.N. Zhang, Journal of Aerospace Power, Vol. 26 (2011) 1274-1281, In Chinese.

15. W.B. Xiao, W.C. Dong, JOURNAL OF NAVAL UNIVERSITY OF ENGINEERING, Vol. 3 (2012) 46-51, In Chinese.

16. Z.P. Shi, T.S. Zhao, M. Li, et al., Chinese Journal of Scientific Instrument, Vol. 33 (2012), pp.1062-1069, In Chinese.

17. F. Chen, Y.M. Xu, et al., Transducer and Microsystem Technologies(2014), In Chinese. 